\title{
RESEARCH ON APPLYING ANALYTIC HIERARCHY PROCESS APPROACH TO TECHNOLOGY ROADMAPPING
}

\author{
Chunyan YANG, Ming YU \\ Dept. Industrial Engineering, \\ Tsinghua University, Beijing, 100084, China \\ yangcy98@mails.tsinghua.edu.cn \\ mingyu@tsinghua.edu.cn \\ Yedong Liao \\ Beijing Eastar Technology Co, Ltd. \\ liaoyedong@tsinghua.org.cn
}

Keywords: Technology roadmapping, AHP, group decision making

\begin{abstract}
Technology roadmapping is a tool for technology planning which has been caused more and more attention in both academic and industrial fields. In this paper, firstly, we summarize the technical problems emerging within technology roadmapping. Then, we demonstrate a methodology based on the Analytic Hierarchy Process (AHP) to address the decision making problems in evaluating technology alternatives in technology roadmap. Finally, we present a process which is easily implemental approach to technology roadmapping.
\end{abstract}

\section{INTRODUCTION}

Today, countries face many challenges in global markets. Products are becoming more complex and, at the same time, more customized. Time-to-market for a new product is shrinking and product life cycle is shorting. Competition is global and fierce, especially from technologically advanced countries. In this circumstance, technology planning is important for directing R\&D activities in order to improve developing countries' capability to access, apply and adapt knowledge or technology know-how, thus staying closer to market demand and reducing the risk of costly investment in R\&D.

Technology roadmapping is an important tool for collaborative technology planning and coordination for corporations as well as for entire industries. It is a needs-driven technology planning process to help identify, select, and develop technology alternatives to satisfy a set of product needs. It brings together a team of experts to develop a framework for organizing and presenting the critical technology-planning information to make the appropriate technology investment decisions and to leverage those investments [1].

As a result of technology roadmapping, a company or an industry can make better investment decisions because it has better information to:

- Identify critical product needs that will drive technology selection and development decisions. 
- Determine the technology alternatives that can satisfy critical product needs.

- Select the appropriate technology alternatives.

- Generate and implement a plan to develop and deploy appropriate technology alternatives.

Among the four tasks, to select the core technology and priority setting is a big challenge because the selection process has various criteria to consider and many interest groups are involved in the process of decision-making. Moreover, it is very difficult to be quantitative.

There are some methodologies to face these problems, AHP is one of them. Analytic hierarchy process (AHP), it allows decision-makers to determine the relative importance of the criteria and alternative solutions for decision making.

Analytic hierarchy process, first developed in 1980 by Thomas L. Saaty, is a technique of multi-criteria decision making. The AHP has been widely accepted in a number of disciplines by academics and practitioners, and applied extensively to solve complex decision-making problems in different areas, such as planning[4], resources evaluation[5], investment decisions[6][7], ranking of enterprises[8], selecting of automobile purchasing model[9], and setting priorities[10]. The strength of the AHP is that it has the capability of both addressing objective criteria and subjective criteria, and incorporating tangible as well as non-tangible factors especially where the subjective judgments of different individuals constitute an important part of the decision process. AHP can also help complex problems to simplify by decomposition and hierarchical form.

In the following part, we will use AHP approach to priority the key factors in technology roadmap in order to select the appropriate technology alternatives.

\section{THE FUNDAMENTALS OF TECHNOLOGY ROADMAP}

A technology roadmap is the document that is generated by the technology roadmapping process. It identifies (for a set of product needs) the critical system requirements, the product and process performance targets, and the technology alternatives and milestones for meeting those targets. In effect, a technology roadmap identifies alternate technology "roads" for meeting certain performance objectives [1]. 


\subsection{The building blocks of technology roadmap}

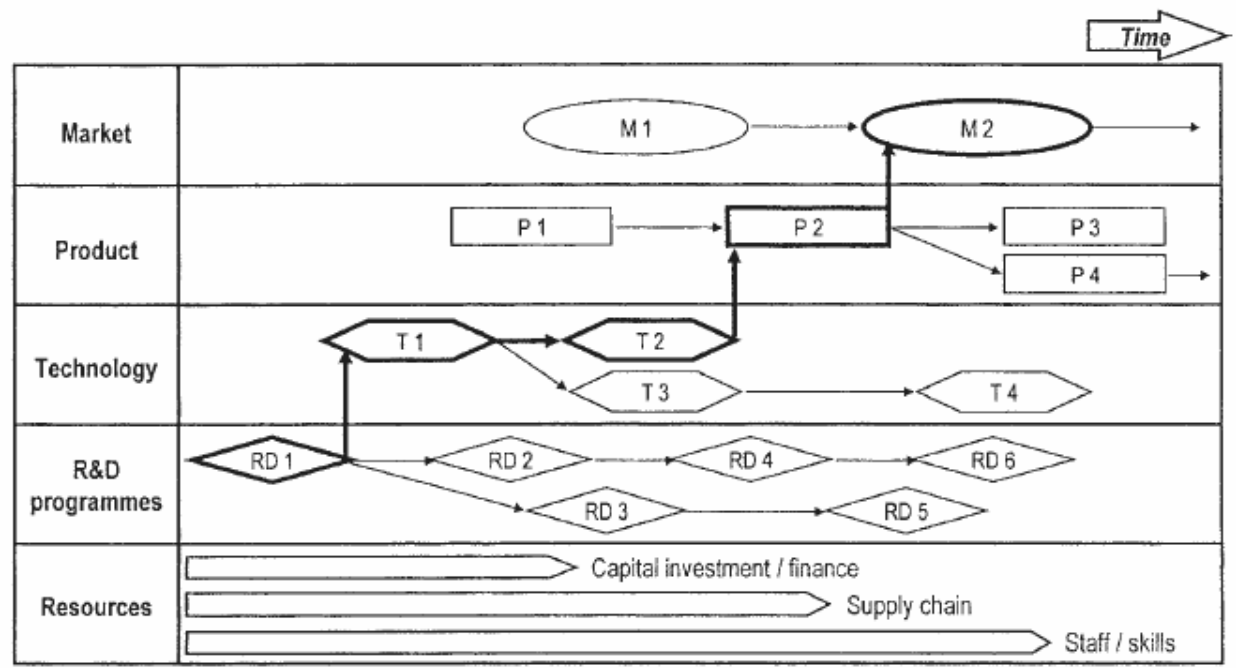

Figure 1 the technology roadmap structure [3]

Technology roadmaps take many forms, although the most general is a multi-layered graphical representation of how technology and product developments link to market opportunities. Figure 1 illustrates the structure of a typical roadmap, comprising a layered chart that shows the linkage between technology, product (or service) and market. The top layer is generally used to represent the market and business drivers, including the purpose of the map (the conditions that have to be met or satisfied). These frequently take the form of trends or milestones, e.g. environmental legislation. The central layer charts the way that these conditions are to be met, i.e. the products, services or capabilities that will be delivered over time. The content of this layer is very variable, depending on the nature of the business activity of the organization being mapped. The lower layer(s) represent technology, and sometimes the resources, that are necessary to deliver the layer above. Choices usually have to be made about which are the important technologies to show on the map, and this requires the use of other prioritization and selection tools [2].

\subsection{The development of technology roadmap}

According to Sandia National Laboratories, which is a government-owned/contractor operated (GOCO) facility, has developed science-based technologies that support United State security, the technology roadmap is developed in the following process [1]:

1) Identify the "product" that will be the focus of the roadmap

The critical step in roadmapping is to get the participants to identify and agree on common product needs that must be satisfied.

2) Identify the critical system requirements and their targets

The critical system requirements provide the overall framework for the roadmap and are the high-level dimensions to which the technologies relate.

3) Specify the major technology areas

These are the major technology areas that can help achieve the critical system requirements for the product.

4) Specify the technology drivers and their targets

At this point, the critical system requirements are transformed into technology-oriented drivers for the 
specific technology areas. These technology drivers are the critical variables that will determine which technology alternatives are selected.

5) Identify technology alternatives and their time lines

Once the technology drivers and their targets are specified, the technology alternatives that can satisfy those targets must be identified. A difficult target may require breakthroughs in several technologies or a technology may impact multiple targets. For each of the identified technology alternatives, the roadmap must also estimate a time line for how it will mature with respect to the technology driver targets. When multiple technologies are being pursued in parallel, decision points need to be identified for when a technology will be considered the winner or when it will be dropped from further consideration.

6) Recommend the technology alternatives that should be pursued In this step, we need to prioritize the technology alternatives.

7) Generate the technology roadmap

During the technology roadmap developing process, how to combine experts' judgments on selecting the appropriate technology alternatives is the key problem. In the following, we focus on integrating AHP approach and group decision-making algorithm to evaluate the technology alternatives (step 6).

\section{METHEDOLOGY: USING AHP TO EVALUATE THE TECHNOLOGY ALTERNATIVES}

Based on the AHP approach and group decision-making algorithm, we can evaluate the technology alternatives step by the following steps:

- To build the decision hierarchy

- To consult experts

- To calculate criteria weight

- To rating the alternatives

We corporate related methods in each method. In the experts consultant step, we choose Delphi method to obtain the experts judgments. In order to combine so many experts' judgments into one synthesis result, we use the geometric mean method in group decision-making.

Step1: Build the decision hierarchy

We can decompose the problem into a graphical hierarchical form to represent goal, criteria, sub-criteria, which build up the core technology evaluation criterion system.

There are some principles in setting up the criteria:

a) Concise principle. The criterion system is decomposed into a clear hierarchical form. Each criterion should be relative independent.

b) Demands principle. The criteria represent the industry development requirements. The core technologies can improvement the traditional and main support industry. This is based on "demand pull”.

c) Economic benefit principle. The core technologies must have high return on investment. The technologies that have high versatility are more important.

d) Scientific principle. In selecting the core technologies, we should analyze the technology trends, follow the technology development rules. This is based on "technology push". 


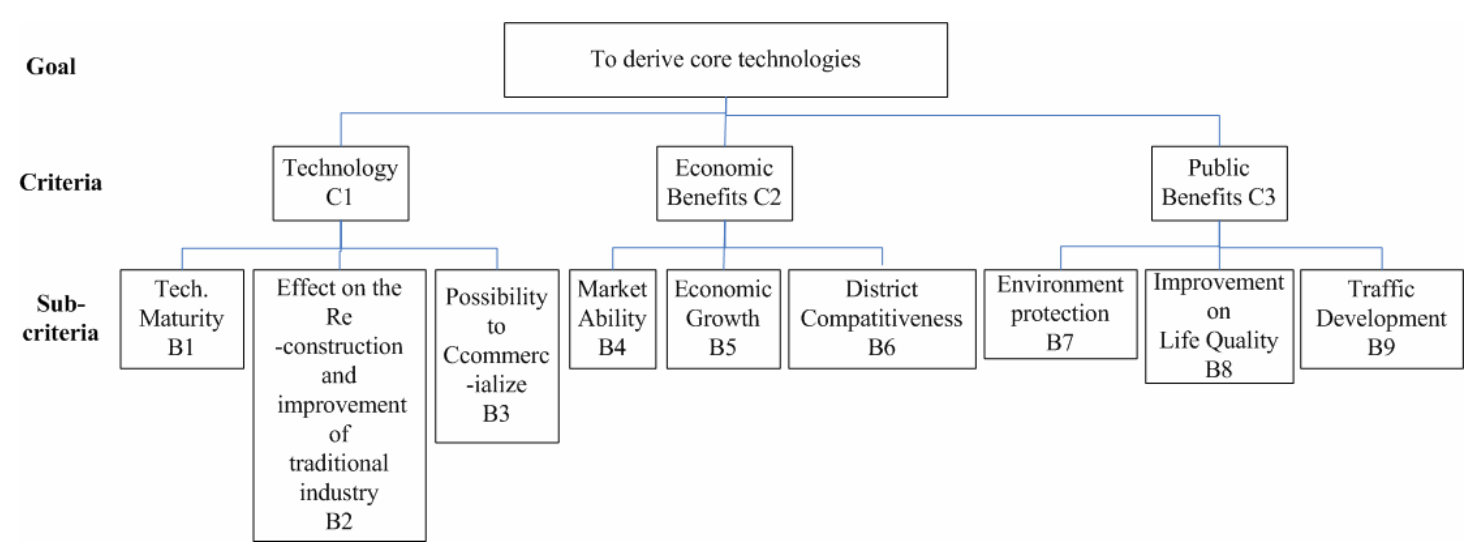

Figure 2 the core technology evaluation criterion hierarchy

According to the principles, we set up the core technology criterion system. See Figure 2. It includes three level of hierarchy, the goal, criteria, and sub-criteria. In the upper level of criteria, $C_{1}, C_{2}, C_{3}$ denote the criteria. In the lower level of criteria (sub-criteria), $B_{1}, B_{2} \ldots B_{9}$ denote the sub-criteria. The next step is that according to the core technology evaluation criterion system, we design the questionnaire, consult the experts.

Step2: Consult experts

We invite 22 experts from different fields, such as industries, universities, research institute, and stakeholders. There are many ways to get the comparison matrix results from experts. The Delphi method is usually applied. The Delphi method aims to obtain the consensus of experts by using a questionnaire survey. This survey method allows experts to express their opinion freely and privately. The key feature of Delphi process is answering the questionnaire over a number of rounds. The questionnaire is sent to a group of selected experts. The results from the first round and the development of the second round questionnaire are supposed to be sent to the experts again in order to make a revised judgment of their previous answers. There is no clear-cut answer as to how many rounds should be undertaken, but at least two rounds should be the minim.

In the first round, we send out 22 expert questionnaires, get back 16 questionnaires. In the second round, we send out 16 expert questionnaires, and pick out 10 questionnaires.

Step3: Calculate criteria weight

We can get the criterion weight in the following way:

1) Get pair-wise comparison matrix from each expert

The matrix is constructed by using a scale of relative importance. The judgments are entered using the fundamental scale of AHP as given in Table 1 .

Table 1Relative importance of factors

\begin{tabular}{ll}
\hline Relative importance & Description \\
\hline 1 & Equal importance of $i$ and $j$ \\
\hline 3 & Moderate importance of $i$ over $j$ \\
\hline 5 & Strong importance of $i$ over $j$ \\
\hline
\end{tabular}




\begin{tabular}{ll}
\hline 7 & Very strong importance of $i$ over $j$ \\
\hline 9 & Absolute importance of $i$ over $j$ \\
\hline $2,4,6,8$ & Intermediate values \\
\hline
\end{tabular}

There are four comparison matrixes. See tables below. They can be one of the questionnaire form.

Respect to goal:

\begin{tabular}{|l|l|l|l|}
\hline Expert number & $\mathrm{C}_{1} / \mathrm{C}_{2}$ & $\mathrm{C}_{1} / \mathrm{C}_{3}$ & $\mathrm{C}_{2} / \mathrm{C}_{3}$ \\
\hline & & & \\
\hline
\end{tabular}

Respect to criteria $\mathrm{A}_{1:}$

\begin{tabular}{|l|l|l|l|}
\hline Expert number & $\mathrm{B}_{1} / \mathrm{B}_{2}$ & $\mathrm{~B}_{1} / \mathrm{B}_{3}$ & $\mathrm{~B}_{2} / \mathrm{B}_{3}$ \\
\hline & & & \\
\hline
\end{tabular}

Respect to criteria $\mathrm{A}_{2}$ :

\begin{tabular}{|l|l|l|l|}
\hline Expert number & $\mathrm{B}_{4} / \mathrm{B}_{5}$ & $\mathrm{~B}_{4} / \mathrm{B}_{6}$ & $\mathrm{~B}_{5} / \mathrm{B}_{6}$ \\
\hline & & & \\
\hline
\end{tabular}

Respect to criteria $\mathrm{A}_{3}$ :

\begin{tabular}{|l|l|l|l|}
\hline Expert number & $\mathrm{B}_{7} / \mathrm{B}_{8}$ & $\mathrm{~B}_{7} / \mathrm{B}_{9}$ & $\mathrm{~B}_{8} / \mathrm{B}_{9}$ \\
\hline & & & \\
\hline
\end{tabular}

2) Using eigenvector to calculate each comparison matrix weight

The comparison matrix from each expert is denoted as $A_{l}=\left[a_{i j, l}\right]_{n \times n}, l=1,2 \ldots, m, m$ is the number of experts. Here, $m=10$ (effective questionnaires)

$A_{l} W=\lambda_{\text {max }} W$, the eigenvector is denoted as $W_{l}$.

$w_{l}=\left(\omega_{1 l}, \omega_{2 l} \ldots \omega_{n l}\right)^{T}, \sum_{i=1}^{n} \omega_{i l}=1, i=1,2 \ldots n$

3) Check the consistency of each comparison matrix.

The consistency index CI is calculated as follows:

$C I=\left(\lambda_{\max }-n\right) /(n-1)$

The random index (RI) for the number of factors is obtained according to Table 2. Then calculate the consistency ratio, $\mathrm{CR}=\mathrm{CI} / \mathrm{RI}$.

Table 2 Random index

\begin{tabular}{|c|c|c|c|c|c|c|c|c|c|}
\hline Number of factors & 1 & 2 & 3 & 4 & 5 & 6 & 7 & 8 & 9 \\
\hline RI & 0.00 & 0.00 & 0.58 & 0.90 & 1.12 & 1.24 & 1.32 & 1.41 & 1.45 \\
\hline
\end{tabular}

4) Using weighted geometric mean to combine group judgments

To combine group judgments and satisfy the reciprocal property from the group in comparing two items in the AHP, one must use the geometric mean.

The composite eigenvector is calculated as following: 
$\bar{\omega}_{i}=\prod_{l=1}^{m} \omega_{l_{i}}^{\lambda_{i}}, i=1,2 \ldots, n$

Here $\lambda_{1}, \lambda_{2}, \ldots . \lambda_{l}$ are the weight of each expert. It is the synthetically quantitative representation of the capability of experts. In brief, we can take $\lambda_{\mathrm{I}}=1 / m, \mathrm{i}=1,2 \ldots m$, so,

$\bar{\omega}_{i}=\prod_{l=1}^{m} \omega_{l_{i}}^{1 / m}, i=1,2 \ldots, n$

Then we can normalize composted eigenvector $w=\left(\omega_{1}, \omega_{2} \ldots \omega_{n}\right)^{T}, \omega_{i}=\frac{\omega_{i}}{\sum_{j=1}^{n} \bar{\omega}_{j}}, i=1,2, \ldots n$

5) Obtain composite criteria weight

The composite weights are obtained by multiplying the relative normalized weight of each factor with its corresponding normalized weight value for each alternative and making summation over all the factors for each alternative.

Step 4: Rating the alternatives

The composite weights are obtained by multiplying the relative normalized weight of each factor with its corresponding normalized weight value for each alternative and making summation over all the factors for each alternative.

$s_{i}=\sum_{j=1}^{n} \omega_{B j} z_{i j}, i=1,2 \ldots n$

$S_{i}$ is synthesis score of the technology $i . \omega_{B j}$ is the weight of each sub-criterion. $z_{i j}$ is the relative score of technology $i$ under sub-criterion $j$. We rank the technologies by score $s_{i}$, and we can get the each technology evaluation result.

\section{CONCLUSIONS}

The traditional technology planning tools include technology forecasting, technology foresight, etc. Technology roadmapping is a new evolution tool for technology planning. Technology roadmapping is critical when the technology investment decision is not straight forward. This occurs when it is not clear which technology alternatives to pursue, and when there is a need to coordinate the development of multiple technologies. The problem in technology roadmapping is how to deal with a complex system with different related techniques, different experts and opinions, different concerns and indices.

The AHP offers a unique and valuable method for the generation and evaluation of technology roadmap. The methodology is based on AHP and group decision-making algorithm. It aims at solving the problem in technology roadmapping. We are applying this methodology to some cases including the technologies investment. We find that the process is easily implemental and offers a relatively quick 
and simple approach to technology roadmapping.

\section{REFERENCES}

[1] Sandia National Laboratories. Fundamentals of Technology Roadmapping. SAND97-0665 Unlimited Release. Printed April 1997

[2] Probert, D.R., Farrukh, C.J.P. and Phaal, R. (2003). Technology roadmapping -developing a practical approach for linking resources to strategic goals. Proceedings of the Institute of Mechanical Engineers, Vol. 217, Part B: J. Engineering Manufacture.

[3] EIRMA. Technology roadmapping:delivering business vision. Working Group Report 52, European Industrial Research Association, Paris, France, 1997

[4] Radash DK, Kwak NK. An integrated mathematical programming model for offset planning. Computers and Operations Research 1998;25(12):1069-83

[5] Zone-Ching Lin, Chu-Been Yang, "Evaluation of machine selection by the AHP method”, Journal of Materials Processing Technology 57 (1996) 253-258.

[6] Bharat A.Jain, Barin N.Nag, "A decision-support model for investment decisions in new ventures”, European Journal of Operational Research 90(1996) 473-486.

[7] Qing Li, Hanif D.Sherali, “An approach for analyzing foreign direct investment projects with application to China's Tumen River Area development”, Computers \& Operations Research 30(2003) 1467-1485

[8] Zoran Babic, Neli Plazibat, "Ranking of enterprises based on multicriterial analysis”, Int.J. Production Economics 56-57 (1998) 29-35

[9] Dae-Ho Byun, “The AHP approach for selecting an automobile purchase model”, Information \& Management 38 (2001) 289-297

[10] Ernest Forman, Kirti Peniwati. Aggregating individual judgments and priorities with the Analytic Hierarchy Process. European Journal of Operational Research 108(1998) 165-169.

[11] Athakorn Kengpol. Design of a decision support system to evaluate the investment in a new distribution centre. Int.J. Production Economics 90 (2004) 59-70 